\title{
Triaxial residual strains in a railway rail measured by neutron diffraction
}

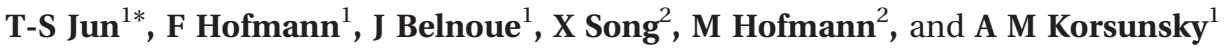 \\ ${ }^{1}$ Department of Engineering Science, University of Oxford, Oxford, UK \\ ${ }^{2}$ FRM II, TU München, Garching, Germany
}

The manuscript was received on 25 March 2009 and was accepted after revision for publication on 1 June 2009.

DOI: $10.1243 / 03093247 J S A 545$

\begin{abstract}
Accumulation of residual stresses in rails during service can contribute to crack initiation and fracture and may result in serious accidents. It is therefore necessary and important to quantify the residual stresses that evolve under repeated rolling contact between wheel and rail. In the present study, triaxial residual strain measurements were performed in a worn British railway rail using neutron diffraction. Localized stress is observed close to the region of contact, showing the asymmetry and complexity of distributions that arise from the non-uniform plastic deformation. Contact-induced plasticity is revealed by the broadening (increase in the full width at half-maximum (FWHM)) of the diffraction peaks.
\end{abstract}

Keywords: residual stress, railway rail, rolling contact fatigue, neutron diffraction, FWHM

\section{INTRODUCTION}

In railway rails, residual stresses are introduced by the repeated action of rolling wheel contact loads. These residual stresses, along with the stresses from manufacturing processes, are known to be a significant component of the total stress that affects the rate of crack initiation and propagation via plasticity-induced damage and microstructural modification. The presence of residual stresses also influences the critical crack size for fast fracture. Even in the absence of macroscopic fracture, microcracking phenomena result in deterioration of the running surface and reduction in the useful rail service life $[\mathbf{1}, \mathbf{2}]$.

The manufacture of a railway rail involves a number of processing steps. First, repeated hot rolling is used to transform a fairly short rectangular steel bloom into a long rail with a specified profile. During this process, the material is not able to support a significant amount of stress, as it is softened by heat [3]. The rail is then left to cool on a walking-beam cooling bed. The varying cooling rates in different regions of the rail also mean that

*Corresponding author: Department of Engineering Sciences, University of Oxford, Parks Road, Oxford OX1 3PJ, UK.

email: tea.jun@eng.ox.ac.uk the cooled rail may become curved and will require straightening by cold rolling or stretching. One of the main results of the cooling process is distortion of the rail, which the roller straightening process corrects. This correction is one of the main sources of residual stresses in a new rail. Finally, the head of the rail is often heat treated for certain uses (e.g. the $350 \mathrm{HT}$ grade) to harden the material in this area so that it can better withstand the high contact stresses in service.

Once in service, the passage of train wheels over the rail introduces non-uniform plastic deformation in the head of the rail close to the running line. After an initial ratcheting period, a shakedown state is reached, and a new residual stress pattern is established in the rail [4]. This is a function of the axle loads, train speeds, gross tonnage, running line position, and, especially in curved sections of the track, the tangential forces.

Previously, residual stress measurements have been carried out using diffraction and other methods to measure the residual elastic strain fields in used and virgin rails [5-7]. In the vicinity of the running surface of the rail head, a layer of compressive stresses resulting from the normal contact load provides some protection against fatigue crack initiation and growth. However, tension exists beneath this layer as a consequence of stress 


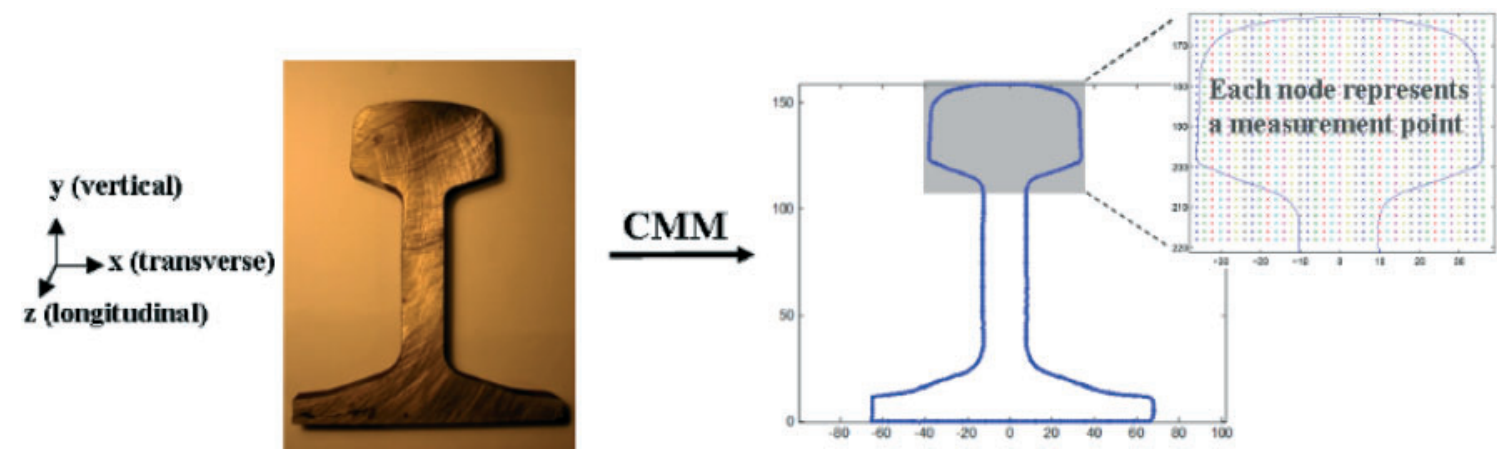

Fig. 1 Specification of the rail sample and the CMM outline image with magnified head region for stress measurement

balance, and these tensile residual stresses in rail heads may eventually promote the growth of any existing cracks that reach this area. It can be seen from the literature $[\mathbf{8}]$ that uncontrolled residual stresses in rails can lead to serious rail accidents, such as the catastrophic failure that occurred in Texas in 1983. It is therefore necessary to quantify the residual stresses for the purposes of improved rail component design and the avoidance of failure in service.

The objective of the present study was to characterize the residual stress distribution through the collection of three-dimensional maps of residual elastic strain in a cross-section of a used British railway rail by neutron diffraction. A thin $(16 \mathrm{~mm}$ thick) transverse slice from the rail was used for strain measurements using neutron diffraction. Evidently, cutting out a slice causes some relaxation of the longitudinal stresses in the rail, so that the stresses measured in the slice are partly relieved compared with those in the full-length rail. However, provided the slicing is carried out in a minimally disturbing way, the results may ultimately be interpreted by means of inverse eigenstrain analysis to determine the underlying permanent inelastic strains [9]. As eigenstrains act as a source of residual stresses, imposing the found distribution onto the full (uncut) model of the rail then allows the threedimensional residual stress state to be reconstructed.

\section{EXPERIMENT}

A $16 \mathrm{~mm}$ thick cross-sectional slice was cut from a section of used 260-grade pearlitic steel railway rail manufactured according to British Railtrack specification RT/CE/S/061 [10]. Note that this grade is different from the more common BS 11:1985 and EN 13674-1:2003 standards (known as grades 113A and $56 \mathrm{E} 1$, with weights of $113 \mathrm{lb} /$ yard and $56 \mathrm{~kg} / \mathrm{m}$ respectively). The key area of interest in this investigation was the head of the rail. The overall sample shape is shown in Fig. 1, where $x$ will also be referred to as the transverse direction, $y$ as the vertical direction, and $z$ as the longitudinal direction. Before carrying out the stress measurements, the outline shape of the rail was found using a coordinate measuring machine (CMM) (Mitutoyo). Although a section of used rail was studied, no significant observable wear in terms of profile change was found. However, it is important to note that some rails become substantially worn during service. In preparation for the experiment, the outline rail section contour was plotted in Matlab, and a grid of points with a spacing of $2 \mathrm{~mm}$ was superimposed to identify the locations of the centres of measurement gauge volumes. The use of relatively large gauge volumes allows good averaging and produces smoother maps well suited to interpretation by inverse eigenstrain analysis.

Residual strain measurements were performed on a Stress-Spec neutron diffractometer at the FRM-II facility in Garching, Germany (TU München), using a constant neutron wavelength of $\lambda=1.6638 \AA$ and a gauge volume of $2 \times 2 \times 2 \mathrm{~mm}^{3}$. Figure 2 shows the experimental strain scanner set-up with identification of the main components: incident beam slits, sample stages, detector, etc.

The diffractometer comprises a highly flexible setup using three different monochromators: Ge (5 11 l), bent silicon Si ( $\left.\begin{array}{lll}4 & 0 & 0\end{array}\right)$, and pyrolitic graphite $\left(\begin{array}{lll}0 & 0 & 2\end{array}\right)$. A two-dimensional position-sensitive ${ }^{3} \mathrm{He}$ detector (PSD), which is a multiwire detector with delay time encoding and with an active area of $200 \times 200 \mathrm{~mm}^{2}$, was used to collect the diffraction patterns. The sample was aligned on the translation stage such that the measurement reference frame coincided with the system of axes assigned in Matlab. Thus, the centroid of each gauge volume could be accurately 


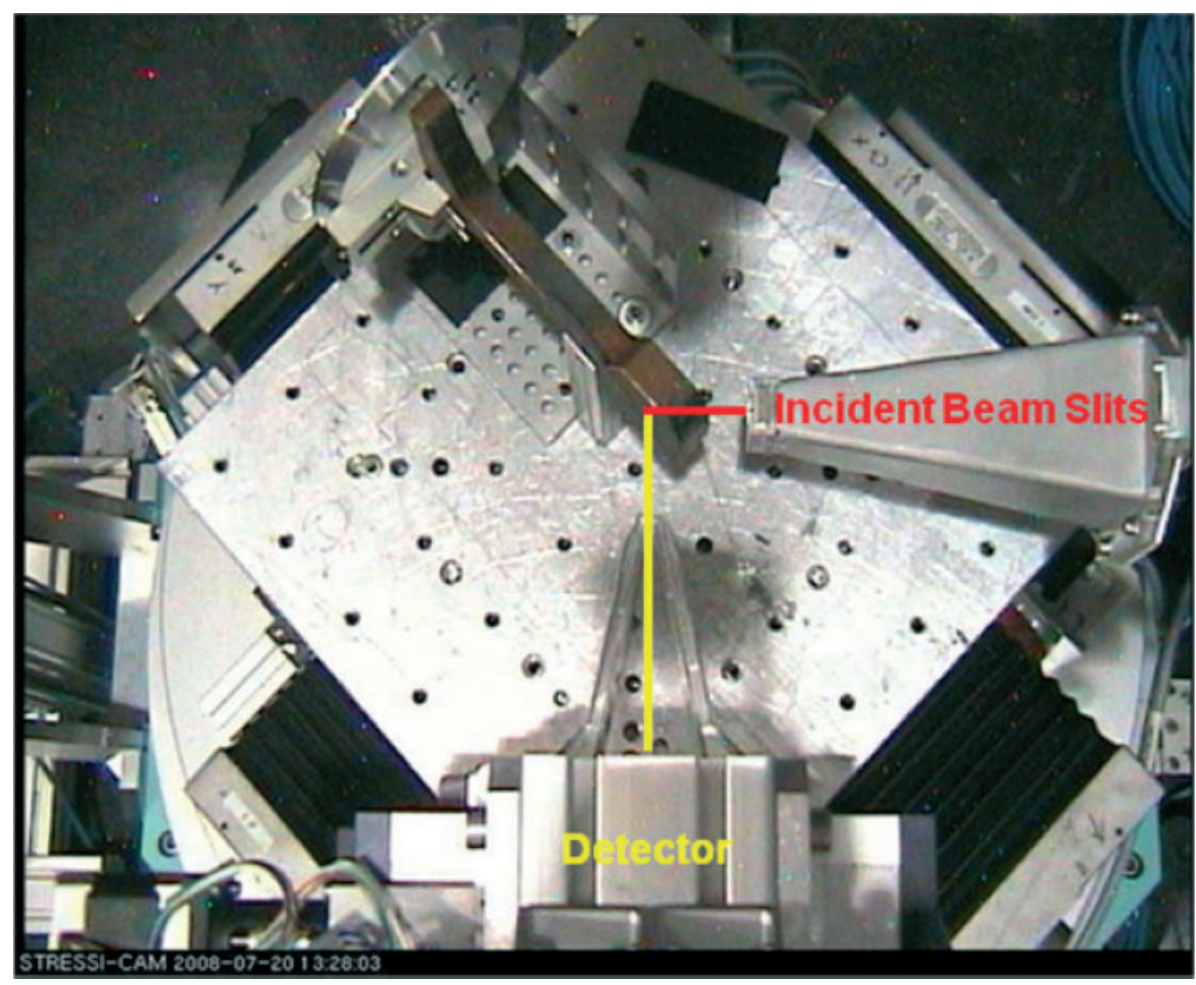

Fig. 2 Experimental set-up at Stress-Spec

lined up with the corresponding measurement node. For measurement of the lattice spacing in the steel, the (2 11 1) reflection was used, as it is generally recommended in the literature for evaluation of the macrostrains in materials with $\alpha$-Fe lattices $[6,7, \mathbf{1 1}$. This is due to the good peak intensity and its relative insensitivity to plastic strain effects. Diffraction patterns were collapsed into line profiles, and peak centre positions were determined by fitting, allowing the shifts of the Bragg peak centres $\Delta \theta$ to be determined [12].

Bragg's law relates the radiation wavelength $\lambda$ to the interplanar lattice spacing $d_{h k l}$ and the scattering angle $\theta: n \lambda=2 d_{h k l} \sin \theta$. Based on this relationship, the elastic strain is determined from the change in the atomic interplanar distances, as compared with the value of $d_{h k l}^{0}$ measured in the unstressed (or reference) condition, $\varepsilon_{h k l}=\left(d_{h k l}-d_{h k l}^{0}\right) / d_{h k l}^{0}$. In the absence of reference strain-free samples, the unstrained lattice parameters $d_{h k l}^{0}$ were obtained from the condition of stress balance across the section, using the $d_{h k l}$ values for three strain directions and based on the assumption of no spatial variation in $d_{h k l}^{0}$ within the sample.

Data analysis was carried out on diffraction patterns obtained at each measurement position in three directions. The strain data obtained were used to calculate the residual stresses using Hooke's law for triaxial stress, i.e.

$$
\begin{gathered}
\sigma_{x}=\frac{E}{(1+v)(1-2 v)}\left[(1-v) \varepsilon_{x}+v\left(\varepsilon_{y}+\varepsilon_{z}\right)\right] \\
\sigma_{y}=\frac{E}{(1+v)(1-2 v)}\left[(1-v) \varepsilon_{y}+v\left(\varepsilon_{z}+\varepsilon_{x}\right)\right] \\
\sigma_{z}=\frac{E}{(1+v)(1-2 v)}\left[(1-v) \varepsilon_{z}+v\left(\varepsilon_{x}+\varepsilon_{y}\right)\right]
\end{gathered}
$$

where $E=200 \mathrm{GPa}$ and $v=0.3$.

\section{RESULTS AND DISCUSSION}

Figure 3 shows the distribution of residual elastic strains in the transverse, vertical, and longitudinal components of the rail slice. In the transverse component, compressive strains can be found at the top of the rail head, while tensile strains exist in the middle region. On the other hand, extensive tensile strains can be found in the vertical component. Not surprisingly, the longitudinal strains are relatively smaller in magnitude than the other components owing to the stress relaxation arising from sectioning. Note, however, that the strain 
(a)

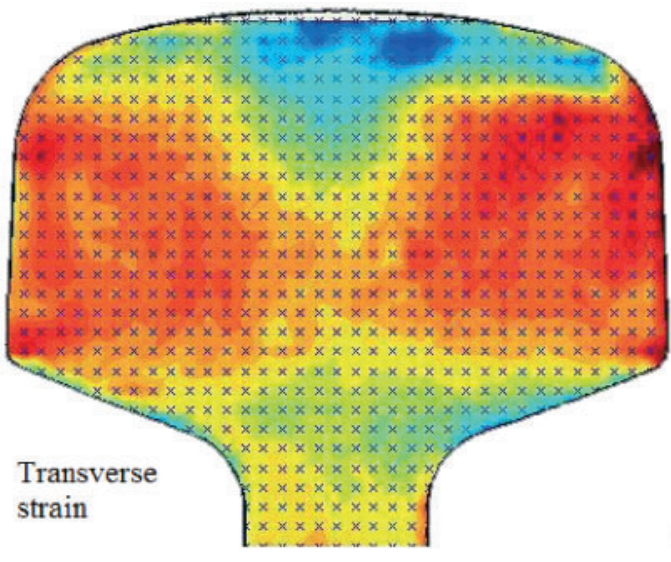

(d)

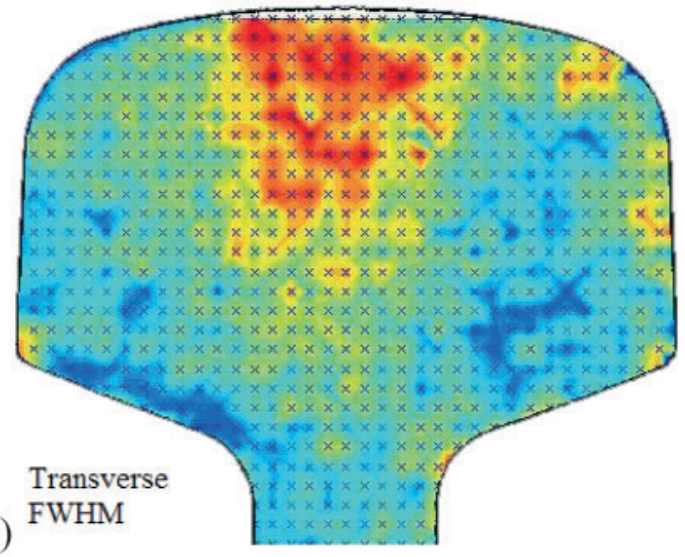

(e)

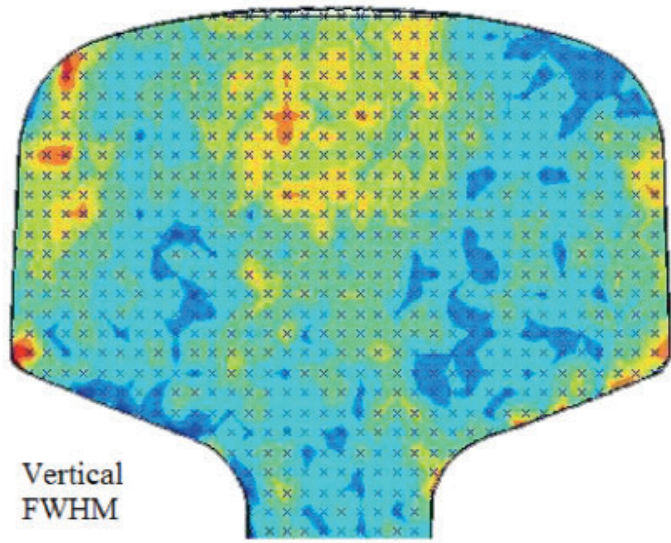

(b)
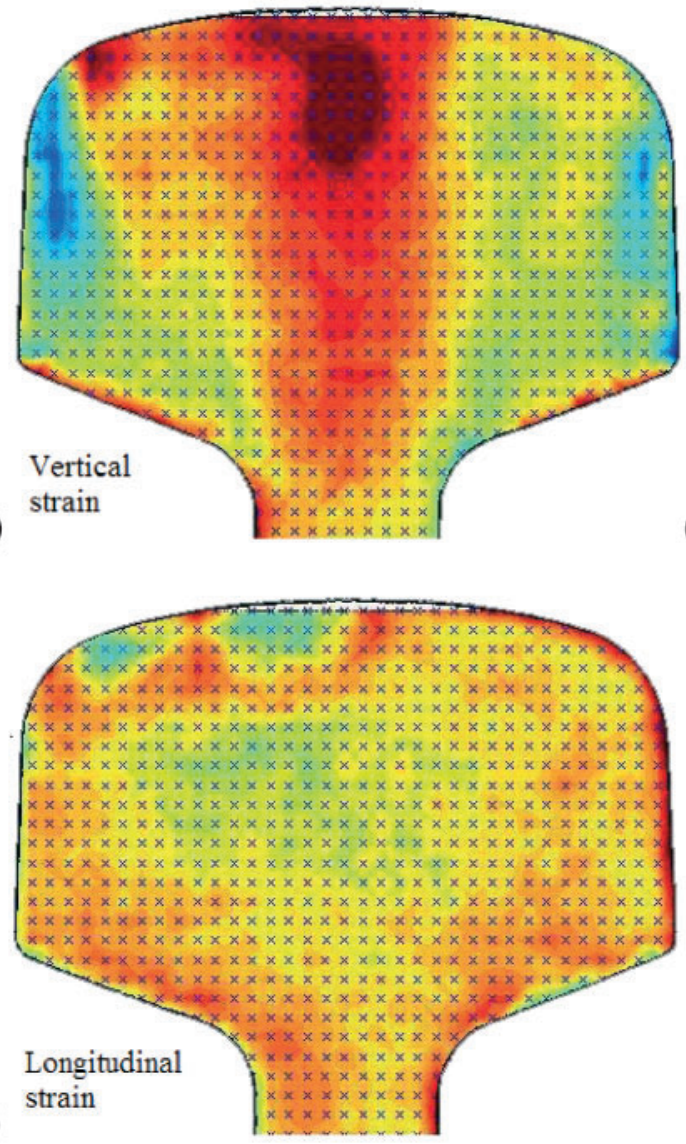

(c)

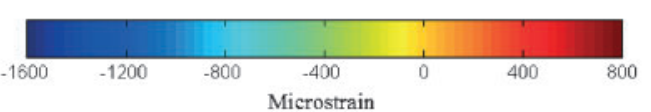

(f)
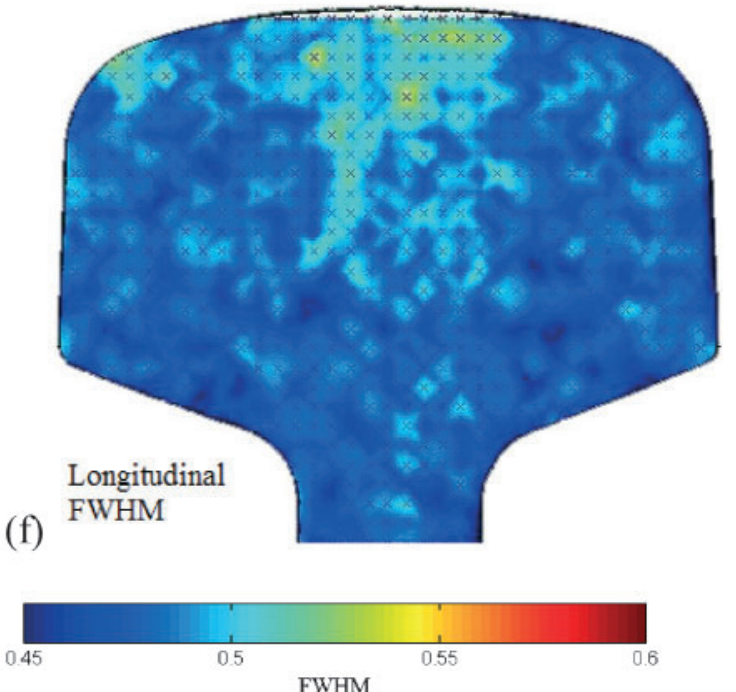

Fig. 3 Distributions of the residual elastic strain components (a)-(c) and FWHM (d)-(f) in the (a), (d) transverse, (b), (e) vertical, and (c), (f) longitudinal, directions

magnitudes are significant and can be taken into account during eigenstrain reconstruction.

The non-uniformly distributed strains near the top of the rail head are a consequence of the passage of trains in service, introducing localized stress and localized plastic deformation. The corresponding contour plots of the FWHM indicate that the diffraction peaks are significantly broadened in the region where non-uniform residual elastic strains exist [11]. Both effects are consequences of the fact that the top of the rail head has undergone plastic deformation. It is also interesting to note the 
difference in the magnitude of FWHM corresponding to different strain components. These are likely to be the consequence of sample microstructure, e.g. different grain dimensions in different directions.

The residual stresses computed using Hooke's law are shown in Fig. 4. The distributions are similar to those of the corresponding strains. The maximum tensile stress exists in the centre of the rail head in the vertical direction, while maximum compressive stress is found below the running surface of the rail head in the transverse direction. This indicates that complex non-uniform stresses exist in the rail head after it has been in service. Figure 5 shows the distribution of the maximum principal stress. Tensile stresses exist mainly in the rail head, apart from the region near the non-contact corner of the rail top surface. This is the consequence of localized force introducing asymmetry of deformation within the rail head.

The significance of the set of results obtained in the present study is that it provides a carefully validated set of experimental measurements that (a) can serve as the basis for advanced models of wheelrail contact and complex material constitutive laws, including kinematic-isotropic cyclic hardening, and (b) are required to predict correctly the long-term response and shakedown behaviour of the rail. Note that, for the purpose of comparison, the rail sectioning exercise can be performed within the simulation, so that the associated stress redistribution can be captured. Alternatively, inverse eigenstrain analysis can be undertaken in order to reconstruct the three-dimensional residual stresses in the full rail. However, this analysis requires the development of suitable representations of the underlying eigenstrains, and will be the subject of a separate study.

\section{CONCLUSION}

Residual stress measurements on a $16 \mathrm{~mm}$ thick transverse slice from a British railway rail were carried out using neutron diffraction. This method was chosen as particularly suitable for the present study owing to its superior penetration into heavier metals. Complex strains and stresses were found in the rail head, and analysis of the FWHM of the corresponding peaks highlighted the fact that these arose from the non-uniform plastic deformation which itself resulted from the repeated passage of trains in service.
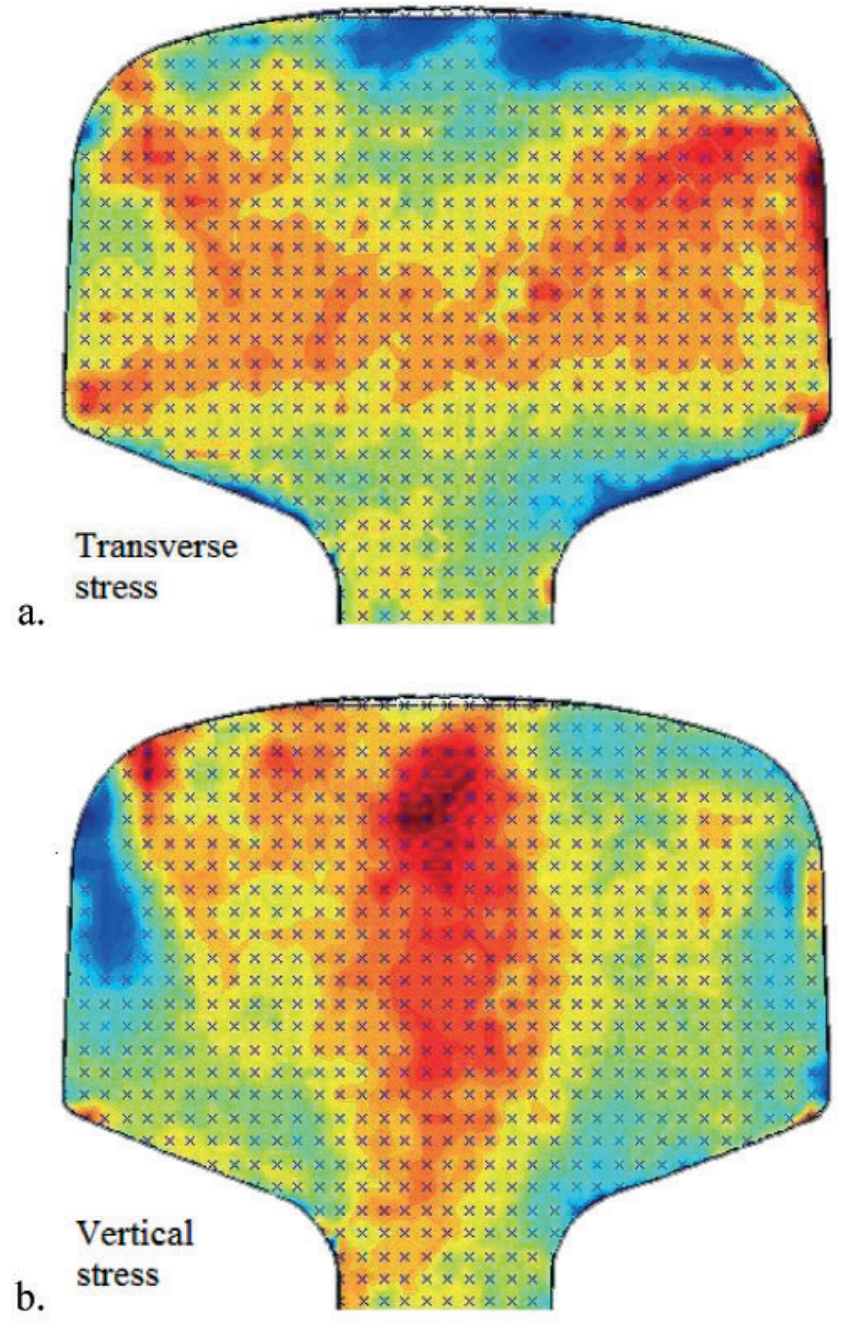

b.
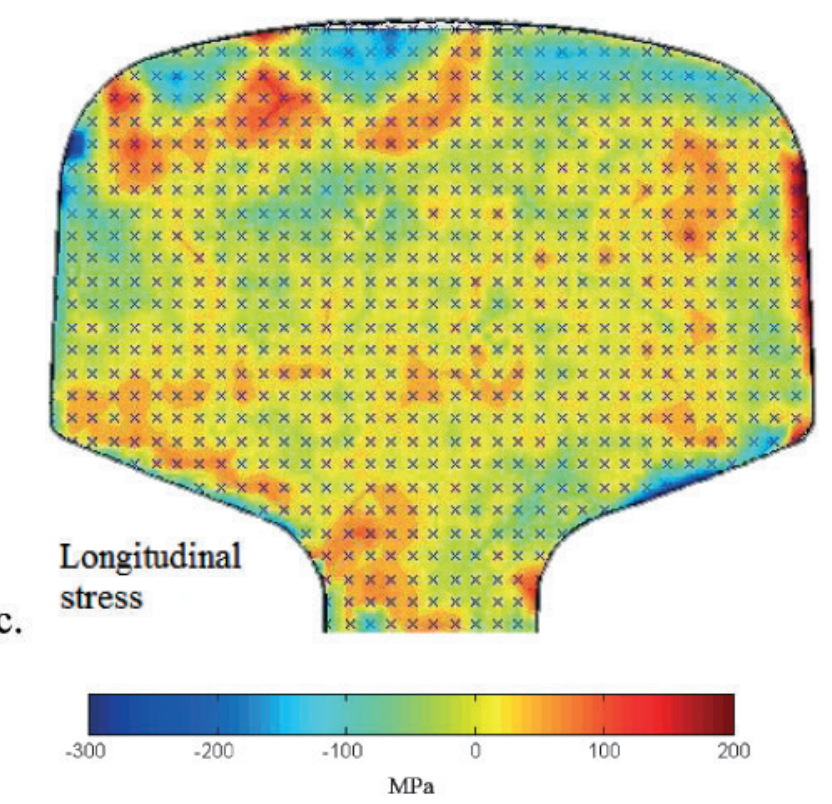

Fig. 4 Distributions of the residual stress components: (a) transverse; (b) vertical; (c) longitudinal 


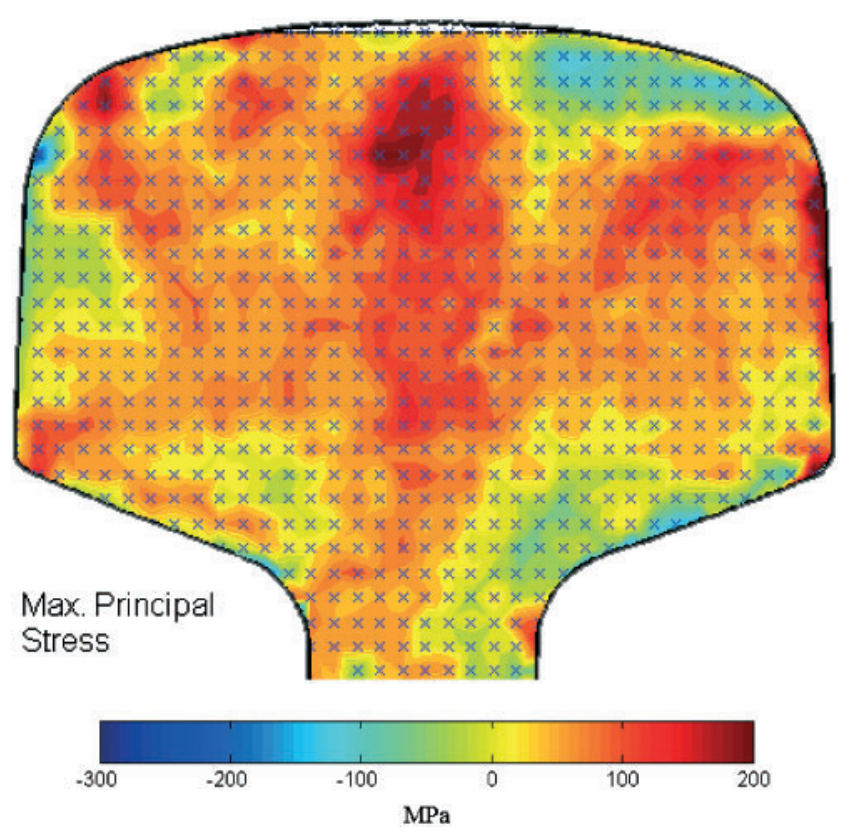

Fig. 5 Distribution of the maximum principal stress

\section{ACKNOWLEDGEMENT}

The authors would like to acknowledge access to the FRM II in Garching, Germany (TU München), through beamtime allocation for experiment 1725 .

(c) Authors 2009

\section{REFERENCES}

1 Orringer, O., Orkisz, J., and Świderski, Z. Residual stress in rails. Vol.1. Field experience and test results, 1992 (Kluwer Academic Publishers, Dordrecht, The Netherlands).

2 Sasaki, T., Takahashi, S., Kanematsu, Y., Satoh, Y., Iwafuchi, K., Ishida, M., and Morii, Y. Mea- surement of residual stresses in rails by neutron diffraction. Wear, 2008, 265, 1402-1407.

3 Basu, J., Srimani, S. L., and Gupta, D. S. Rail behaviour during cooling after hot rolling. J. Strain Analysis, 2004, 39(1), 15-24.

4 Ringsberg, J. W. Cyclic ratchetting and failure of a pearlitic rail steel. Fatigue and Fracture Engng Mater. and Struct., 2000, 23(9), 747-758.

5 Stefanescu, D., Truman, C. E., and Smith, D. J. An integrated approach for measuring near-surface and subsurface residual stress in engineering components. J. Strain Analysis, 2004, 39(5), 483497. DOI: $10.1243 / 030932404189652$.

6 Webster, P. J., Wang, X., Mills, G., and Webster, G. A. Residual stress changes in railway rails. Phys. $B, 1992$, 180-181, 1029-1031.

7 Kelleher, J., Prime, M. B., Buttle, D., Mummery, P. M., Webster, P. J., Shackleton, J., and Withers, P. J. The measurement of residual stress in railway rails by diffraction and other methods. J. Neutron Diffraction, 2003, 11(4), 187-193.

8 Orringer, O. and Tong, P. Investigation of catastrophic failure of a premium-alloy railroad rail. In Fracture problems in the transportation industry: Proceedings of a section held in conjunction with the ASCE Convention, Detroit, MI, 1985, pp. 62-79.

9 Korsunsky, A. M., Regino, G. M., and Nowell, D. Variational eigenstrain analysis of residual stresses in a welded plate. Int. J. Solids and Struct., 2007, 44, 4574-4591.

10 Railtrack Line Specification RT/CE/S/061, Issue 1, King's Cross Station, London, 1996.

11 Reimers, W., Pyzalla, A. R., Schreyer, A., and Clemens, H. Neutrons and synchrotron radiation in engineering materials science, 2008 (Wiley-VCH, Weinheim, Germany).

12 Hofmann, M., Schneider, R., Seidl, G. A., RebeloKornmeier, J., Wimpory, R. C., Garbe, U., and Brokmeier, H.-G. The new materials science diffractometer Stress-Spec at FRM-II. Phys. B, 2006, 385-386, 1035-1037. 